\title{
A Mixed-Mode Synchronous Mirror Delay Insensitive to Supply and Load Variations
}

\author{
CHIH-HAO SUN AND SHEN-IUAN LIU \\ Department of Electrical Engineering \& Graduate Institute of Electronics Engineering, \\ National Taiwan University, Taipei, Taiwan 10617, ROC \\ E-mail:lsi@cc.ee.ntu.edu.tw
}

Received April 10, 2002; Revised January 8, 2003; Accepted February 14, 2003

\begin{abstract}
A digital synchronous mirror delay combined with an analog delay-locked loop (DLL) is introduced. Under the influence of process, voltage, temperature, and load variations, the conventional digital synchronous mirror delay could not compensate the static phase error because of its digital type and open loop by nature. The proposed circuit can compensate the delay mismatch between the output buffer and the inner stage, which is caused by the different loading conditions. It can improve the noise immunity from supply variations. Moreover, because of the tracking property of the DLL, the static phase error and jitter could also be reduced. The proposed circuit has been fabricated by a CMOS $0.35-\mu \mathrm{m}$ one-poly four-metal process and the whole chip area is $1.47 \times 1.07 \mathrm{~mm}^{2}$ including I/O pad peripherals. The measured peak-to-peak jitter is $16.4 \mathrm{ps}$ at supply voltage of $3.3 \mathrm{~V}$ and frequency of $300 \mathrm{MHz}$. The power consumption of the entire chip is $16.5 \mathrm{~mW}$ for analog part and $84 \mathrm{~mW}$ for digital part. The comparisons between the proposed circuit and the conventional digital synchronous mirror delay are also demonstrated.
\end{abstract}

Key Words: synchronous mirror delay, delay-locked loop, static phase error, jitter

\section{Introduction}

Phase-locked loops (PLL) and delay-locked loops (DLLs) are often used for synchronization in the highspeed digital systems. The recursive feedback adjustment property of the PLLs and DLLs makes it possible to refine the static phase error and the jitter performance. However, the long locking cycles will be needed during the lock-in acquisition process. When the fast locking requirement is needed, hundreds of cycles lock time may be unacceptable [1-3]. In other words, PLL and DLL are clumsy at initial acquisition process and agile at their deskew ability when the phase difference between input and output clock is getting small.

Synchronous Mirror Delay (SMD) [1] is a digital type and non-feedback structure. It could lock the phases of the output clock signal and the input clock signal in only 2 clock cycles. However, any variations caused by process, voltage, temperature, load (PVTL) would affect the static phase error and jitter performance of the output clock signal. According to our simulations, the phase error between the input and output clock signals is $100-200$ ps for $\pm 10 \%$ supply voltage or several pf loading capacitance variations. Moreover, because of the digital delay cells used in the conventional digital SMD (DSMD), there existed a minimum gate delay, i.e. limited phase error, when two clocks are synchronous. The conventional DSMD has no capability to detect and reduce the phase difference because of its open loop structure.

In this paper, we propose a mixed-mode SMD (MSMD) to take the advantages of DSMD with 2-cycle lock time and DLL with low static phase error and jitter performance to be insensitive to supply and load variations. Section 2 reviews the operation of the various kinds of SMDs and their problems. Section 3 discusses the proposed MSMD and its circuits. Section 4 shows the experimental results. Conclusions would be given in Section 5.

\section{Conventional SMDs}

A DSMD [1] is shown in Fig. 1, which includes an input buffer, an output buffer, a delay monitor circuit (DMC), a forward delay array (FDA), a monitor control 


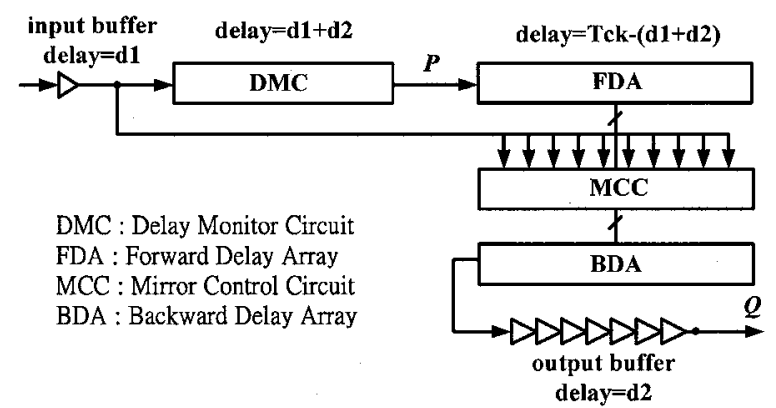

Fig. 1. Conventional synchronous mirror delay [1].

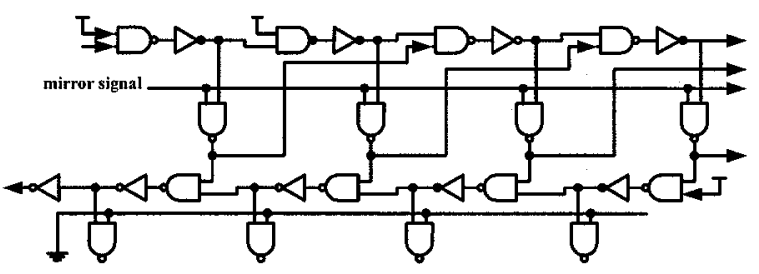

Fig. 2. The schematic of FDA, MCC, and BDA.

circuit (MCC) and a backward delay array (BDA). Assume that the delay of input buffer is $\mathrm{d} 1$ and the delays of output buffer and DMC are $\mathrm{d} 2$ and $\mathrm{d} 1+\mathrm{d} 2$, respectively. When DSMD is activated, the first clock signal goes through input buffer, DMC, and then FDA. The schematics of FDA, MCC, and BDA are shown in Fig. 2. NAND-typed logic gates form the delay array and additional NAND gates are added to match the loading conditions between FDA and BDA. The delay duration of the first clock signal in the FDA is controlled by the second clock signal from the input buffer. The delay between the first and the second clock signals is the delay sum of DMC and FDA. Since the delay of DMC is $\mathrm{d} 1+\mathrm{d} 2$ and the clock period is $\mathrm{T}_{\text {ck }}$, the delay duration of the first clock signal in the FDA is $\mathrm{T}_{\mathrm{ck}}-(\mathrm{d} 1+\mathrm{d} 2)$. Then, the first clock signal continuously goes through the BDA with the same delay of FDA and the output buffer. To summarize the results, the total delay of the first clock signal through a DSMD is $\mathrm{d} 1+(\mathrm{d} 1+\mathrm{d} 2)+\left(\mathrm{T}_{\mathrm{ck}}-\mathrm{d} 1-\mathrm{d} 2\right)+\left(\mathrm{T}_{\mathrm{ck}}-\mathrm{d} 1-\mathrm{d} 2\right)+$ $\mathrm{d} 2=2 \mathrm{~T}_{\text {ck }}$.

From the above descriptions, there is an important assumption that the delay of DMC should be equal to the delay sum of input buffer and output buffer. Since the output node, Q, in Fig. 1, is often connected to the I/O pad or the capacitive load directly. The loading capacitance of node Q would be higher than that of node $\mathrm{P}$, thus the amount of delay would not equal. On

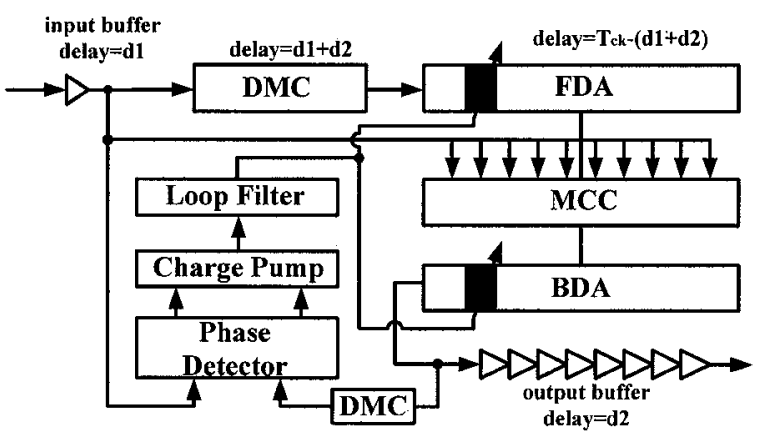

Fig. 3. Mixed-mode DLL [7].

the other hand, the layouts of FDA and BDA should be carefully designed; otherwise, the delays of FDA and BDA could also be different. Besides, the minimum gate delay of digital delay cells in FDA and BDA would affect the minimum static phase error. Moreover, under the influence of the power supply variations, a DSMD could not detect the phase error and neither reduces the phase error.

Many remedies [2-7] had been proposed to improve the performance of a DSMD. However, analog SMDs [4-6] will induce the additional delay from the analog comparator [5] and the problems of the frequencydependent delays [5]. Moreover, analog SMDs need the matched current sources and capacitors. A mixed-mode DLL (MDLL) [7] is shown in Fig. 3, which consists of voltage-controlled unit delays (VCUs) [7] and a chain of fixed delay cells in FDA and BDA. Although MDLL has analog VCUs in the delay array to obtain the necessary delay resolution, it could not sense the loading changes of the output buffer. For any changes of the load, it would affect the delay time of the output buffer. Thus, the delay sum of input buffer and output buffer will differ from the delay of DMC for digital and ana$\log$ SMDs [1-7]. This would go against the original assumption $[1,2]$. When the supply voltage changes or has noise interferences, similar effects will also appear. To improve the static phase error and jitter performance, the proposed MSMD will improve the DSMD to be insensitive to the supply and load variations by using a DLL.

\section{Proposed MSMD}

The proposed mixed-mode SMD (MSMD) is shown in Fig. 4, which consists of a digital SMD, an analog 


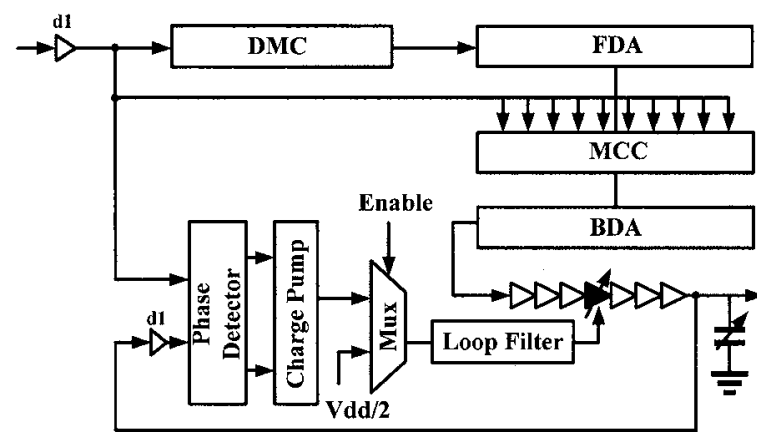

Fig. 4. Proposed mixed-mode synchronous mirror delay.

DLL and a multiplexer. This DLL is composed of a phase detector, current pump, loop filter, and voltagecontrolled delay units used as the part of the output buffer. To compare the performance of the proposed MSMD and the conventional DSMD, the enable signal, as shown in Fig. 4, is used to enable the analog DLL to be a MSMD or disable it to be a DSMD. The operation of the proposed MSMD will be described as follows:

\subsection{Operation of MSMD}

First, the input clock signal would go through the input buffer, DMC, FDA, MCC, BDA, and output buffer in the DSMD. And the output clock signal would be roughly synchronized with the input clock signal within 2-clock cycles. Then, the phase detector compares the phase error between the output clock and the input clock. The detected phase error would generate a control signal through current pump and loop filter to control the dealy of the voltage-controlled delay units in the output buffer. It will reduce the static phase error and improve the jitter performance. The proposed circuit could not only possess the fast lock property of a DSMD but also the fine deskew ability of a DLL.

It's worthy to mention that there is a difference between the MDLL [7] and our proposed MSMD. In the MDLL [7], the voltage-controlled delay units are added in FDA and BDA, its major concern is to eliminate the minimum gate delay of the digital delay cell and improve the jitter performance with the help of the analog loop. It does not consider the load of output signal, which is usually more heavy-loaded than the inner stage. In our proposed MSMD, however, the voltagecontrolled delay unit is added in the output buffer to adjust its delay duration corresponding to a given loading. By the way, the minimum deskewed resolution limit, which is reflected on the static phase error, could also be eliminated through the compensated delay of the voltage-controlled delay units added in the output buffer. Besides, in our proposed MSMD, only an additional input buffer delay is added in the feedback loop, the die area would be saved compared to the MDLL [7], which adds additional DMC in the feedback loop.

\subsection{Delay Locked Loop}

One of the critical building blocks for a DLL is the phase detector (PD). Figure 5 shows the schematic of the dynamic logic type PD [8]. It is composed of two symmetric half circuits that have only six transistors with small parasitic inherently. Compared to the conventional phase detector, the dynamic PD can overcome the speed limitation and reduce the dead zone. The short feedback path and dynamic operation would allow the phase comparison with high precision in the high frequency operation. The current pump used in this DLL is the current-steered type current pump using current mirrors. Figure 6 shows the schematic of the current-steered current pump [9]. The voltagecontrolled delay units and its bias circuit are shown in Fig. 7 [10]. By varying the control voltage, both the trigger points and the output time constant of the delay cells would be changed, thus shifting the phase of the input signal.

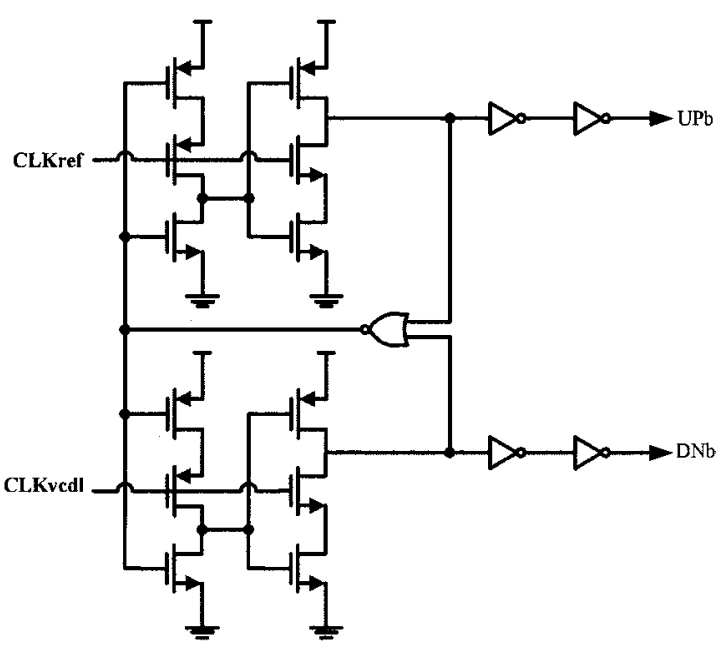

Fig. 5. Dynamic phase detector. 


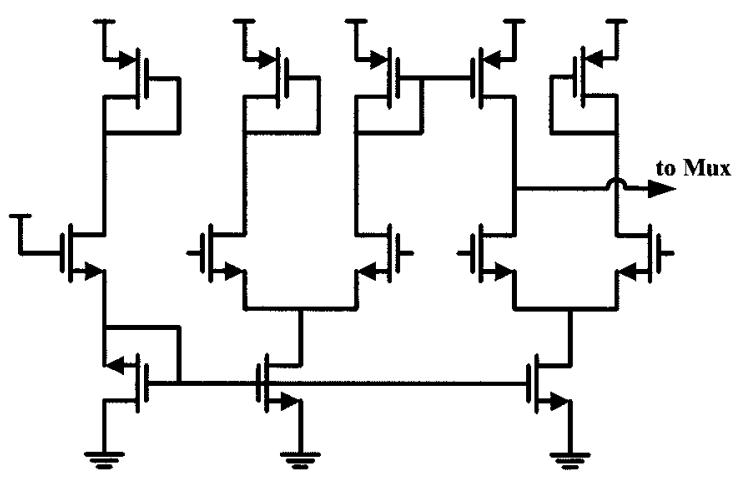

Fig. 6. Current-steered current pump.

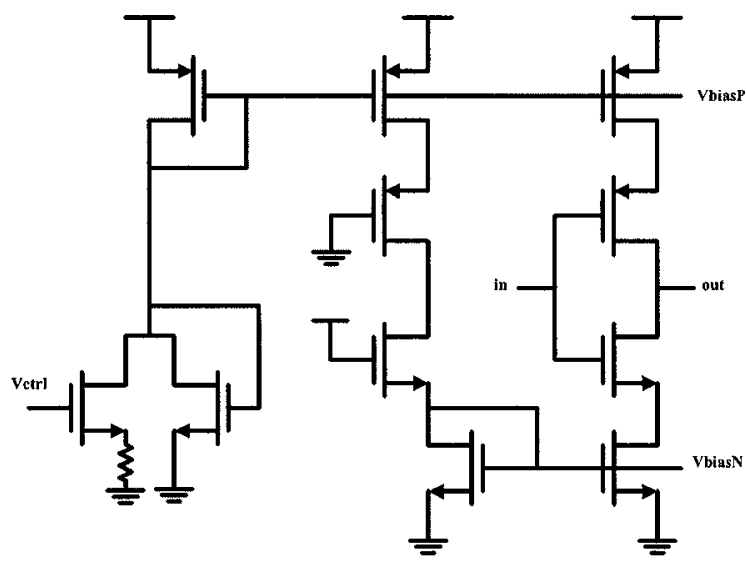

Fig. 7. Voltage-controlled delay unit and its bias circuit.

\section{Experimental Results}

The proposed MSMD has been fabricated in a CMOS $0.35-\mu \mathrm{m}$ one-poly four-metal process. The total die area including I/O peripherals is $1.47 \times 1.07 \mathrm{~mm}^{2}$, as shown in Fig. 8. Figure 9 shows the static phase

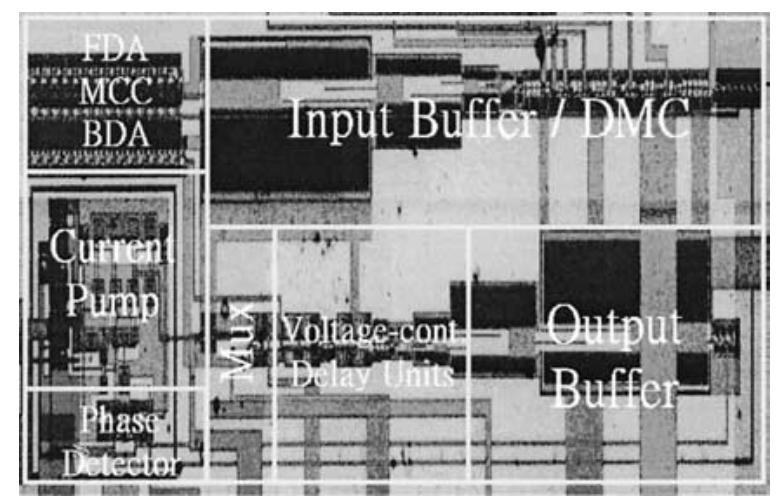

Fig. 8. Die photograph of MSMD.

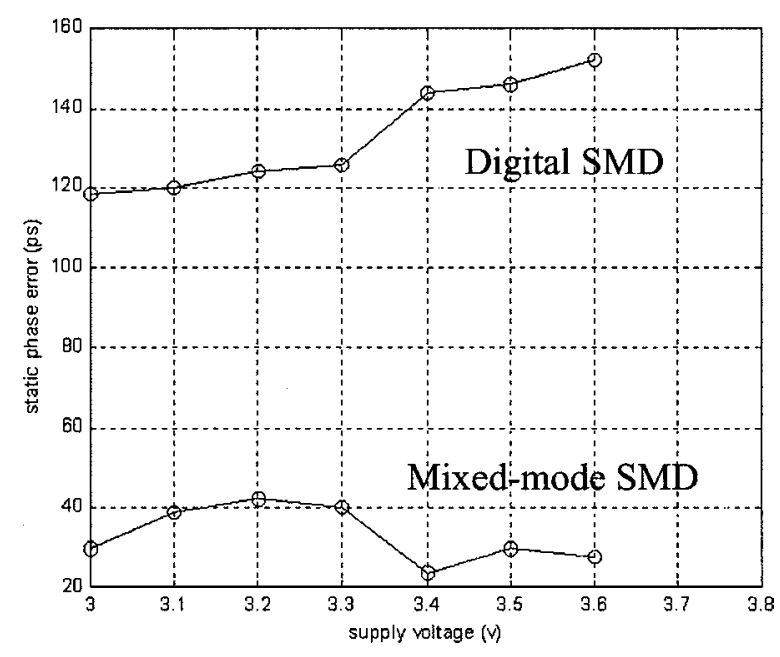

Fig. 9. Static phase error vs. supply voltage variations.

errors of DSMD and MSMD when the power supply deviates from the normal $3.3 \mathrm{~V}$ with $\pm 10 \%$ variations. The results show that when the power supply voltage is increased, the static phase error of DSMD would be getting worse, while the deskew ability of MSMD would track to the input clock signal and maintain the static phase error at an average value around 33 ps. Figure 10 shows the peak-to-peak jitters of DSMD and MSMD, accompanied with the power supply variations. The results reveal that MSMD could have better jitter performance than DSMD under the variations of power supply. Figure 11 shows the static phase errors of DSMD and MSMD, accompanied with variations

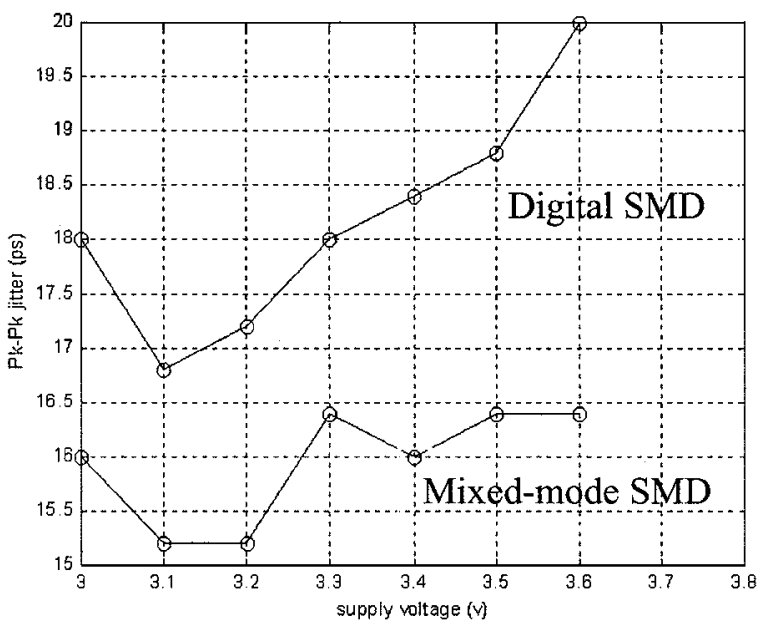

Fig. 10. Peak-to-peak jitter vs. supply voltage variations. 


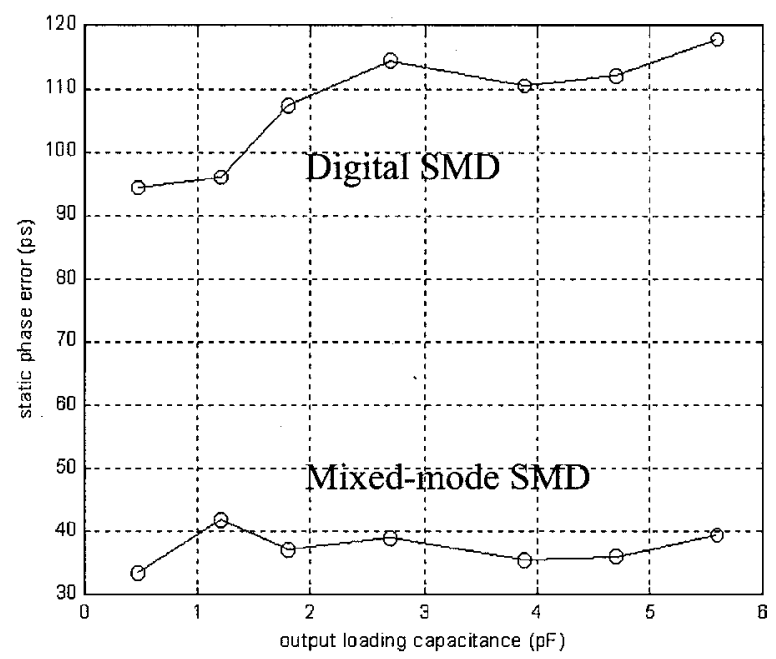

Fig. 11. Static phase error vs. output loading variations.

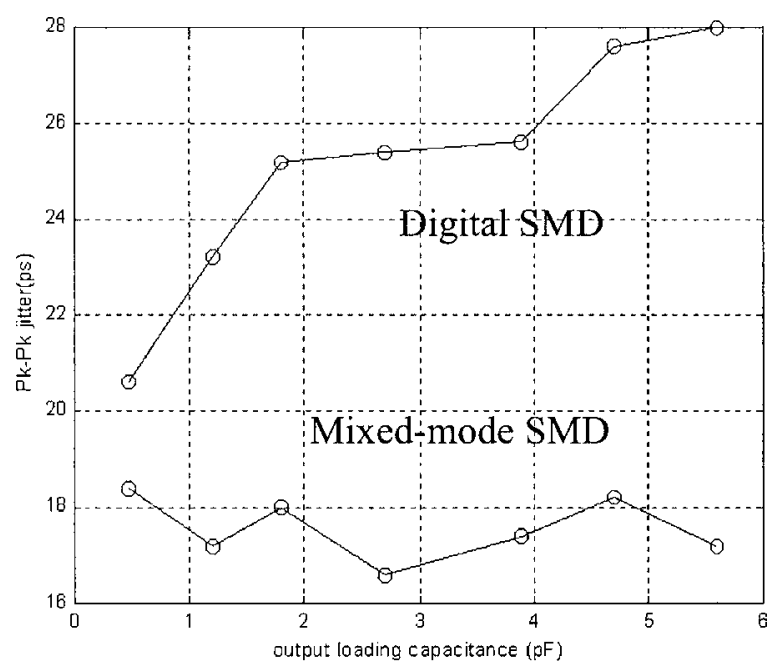

Fig. 12. Peak-to-peak jitter vs. output loading variations.

of output loading capacitance. Based on the measurement results, MSMD could provide deskewed ability better than that of DSMD. Figure 12 shows the peak-topeak jitters of DSMD and MSMD, accompanied with different output loading capacitances. The result verifies that MSMD could have better jitter performance than DSMD when the output load increased. Figure 13 shows the measured jitter histogram of the output clock. The measured rms jitter and peak-to-peak jitter are 1.96 ps and $16.4 \mathrm{ps}$, respectively. Compared with the MDLL [7], the results show that the proposed MSMD could achieve better jitter performance.

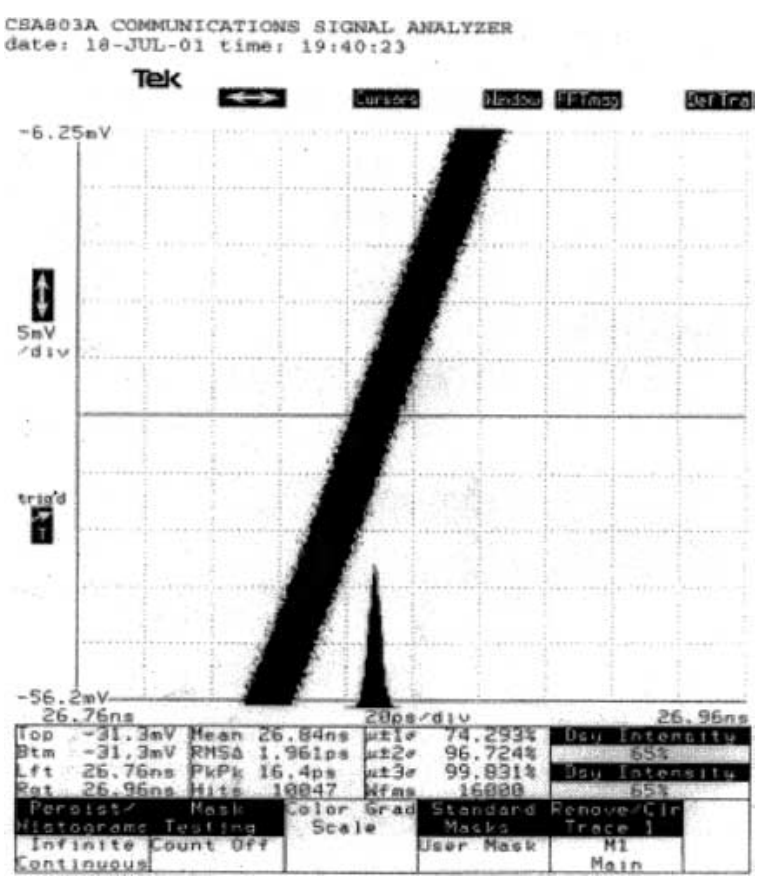

Fig. 13. Measured jitter histogram of the output clock.

\section{Conclusions}

A MSMD is proposed to meet the requirements of immunity to supply and load variations. The performance is summarized in Table 1. The power supply is measured from 3.0 to $3.6 \mathrm{~V}$ and the output loading capacitance is measured toward $5.6 \mathrm{pF}$, excluded the loading effect of the PCB trace and the active probe, and the

Table 1. Performance summary of the proposed MSMD.

\begin{tabular}{ll}
\hline Process & \multicolumn{1}{c}{ CMOS 1P4M TSMC0.35 $\mu \mathrm{m}$} \\
\hline Operating voltage & 3.0-3.6 V @300 MHz \\
Output loading & $0.47-5.6 \mathrm{p} @ 300 \mathrm{MHz}$ \\
Frequency range & 280-320 MHz \\
Static phase error & Min.23.6 ps @MSMD, 3.4 V, 300 MHz \\
& Max.42.0 ps @MSMD, 3.2 V, 300 MHz \\
& Min. 118.4 ps @SMD, 3.0 V, 300 MHz \\
& Max. 152.0 ps @SMD, 3.6 V, 300 MHz \\
RMS jitter & $1.961 \mathrm{ps} @ 3.3 \mathrm{~V}, 300 \mathrm{MHz}$ \\
Peak-to-peak jitter & $16.4 \mathrm{ps} @ 3.3 \mathrm{~V}, 300 \mathrm{MHz}$ \\
Power & Digital $84 \mathrm{~mW} @ 300 \mathrm{MHz}$ \\
& Analog $16.5 \mathrm{~mW} @ 300 \mathrm{MHz}$ \\
Chip area & $1470 \mu \mathrm{m} * 1071 \mu \mathrm{m}$
\end{tabular}


operating frequency range is $280-320 \mathrm{MHz}$. The peakto-peak jitter performance of MSMD could be as low as 16.4 ps under $\pm 10 \%$ power supply deviations, and $18.5 \mathrm{ps}$ under the variations of $5.6 \mathrm{pF}$ output loading capacitance. The results could show that MSMD could provide better jitter performance than DSMD under the variations of supply voltages or output capacitive load.

\section{References}

1. T. Saeki et al., "A 2.5-ns clock access, 250-MHz, 256-Mb SDRAM with synchronous mirror delay." IEEE J. Solid-State Circuits, vol. 31, pp. 1656-1668, 1996.

2. T. Saeki, H. Nakamura, and J. Shimizu, "A 10 ps jitter 2 clock cycle lock time CMOS digital clock generator based on an interleaved synchronous mirror delay scheme." IEEE Symposium on VLSI Circuits, pp. 109-110, 1997.

3. T. Saeki, K. Minami, H. Yoshida, and H. Suzuki, "The direct skew detect synchronous mirror delay (Direct SMD) for ASICs," in Proceedings of the IEEE Custom Integrated Circuits Conference, 1998, pp. 511-514.

4. D. Shim, D.Y. Lee, S. Jung, C.H. Kim, and W. Kim, "An analog synchronous mirror delay for high-speed DRAM application." IEEE J. Solid-State Circuits, vol. 34, pp. 484-493, 1999.

5. J.S. Chae, D. Kim, and D.M. Kim, "Wide range single-waypumping synchronous mirror delay." Electronics Letters, vol. 36, pp. 939-940, 2000.

6. S.J. Jang, Y.H. Jun, J.G. Lee, and B.S. Kong, "ASMD with duty cycle correction scheme for high-speed DRAM." Electronics Letters, vol. 37, pp. 1004-1006, 2001.

7. J.J. Kim, S.B. Lee, T.S. Jung, C.H. Kim, S.I. Cho, and B. Kim, "A low-jitter mixed-mode DLL for high-speed DRAM applications." IEEE J. Solid-State Circuits, vol. 35, pp. 1430-1436, 2000.

8. S. Kim et al., "A 960-Mb/s/pin interface for skew-tolerant bus using low jitter PLL.” IEEE J. Solid-State Circuits, vol. 32, pp. 691-700, 1997

9. J.G. Maneatis, "Low-jitter process-independent DLL and PLL based on self-biased techniques." IEEE J. Solid-State Circuits, vol. 31, no. 11, pp. 1723-1732, 1996.

10. I.M. Filanovsky and H. Baltes, "CMOS Schmitt trigger design." IEEE Trans. on Circuits and System: Part-I, vol. 41, no. 1, pp. 46-49, 1994.

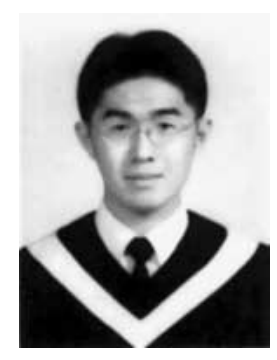

Chih-Hao Sun was born in Taipei, Taiwan, on Sept. 21, 1978. He received his B.S.and M.S. degrees in the Dept. of Electrical Engineering, National Taiwan University, in 2000 and 2002, respectively. Now, He is studying for his Ph.D. degree in the University of Michigan, Ann Arbor.

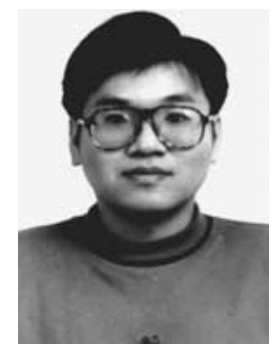

Shen-Iuan Liu was born in Keelung, Taiwan, R.O.C., in 1965. He received the B.S. and Ph.D. degrees in electrical engineering from National Taiwan University, Taipei, Taiwan, in 1987 and 1991, respectively. During 1991-1993, he served as a Second Lieutenant in the Chinese Air Force. During 1991-1994, he was an Associate Professor in the Department of Electronic Engineering of National Taiwan Institute of Technology. He joined in the Department of Electrical Engineering, National Taiwan University, Taipei, Taiwan, in 1994, where he has been a Professor since 1998. His research interests are in analog and digital integrated circuits and systems. He is a senior member of IEEE. 